\title{
THEORIE UND RECHTSVERGLEICHENDE ASPEKTE DER WIRTSCHAFTSINTEGRATION ZWISCHEN ENTWICKLUNGSLÄNDERN
}

\author{
Von E. U. Petersmann
}

1. Regionale Wirtschaftsintegration zwischen Entwicklungsländern: vergleichende entwicklungspolitische Erfahrungen

Seit den raschen Anfangserfolgen der EWG und EFTA und parallel zur europäischen Regionalintegration sowie zur „,internationalen sozialistischen Arbeitsteilung“ zwischen den COMECON-Ländern besteht in allen unterentwickelten Erdteilen eine allgemeine Tendenz zur regionalen Wirtschaftsintegration und damit zugleich eine Tendenz von der internationalen zur interregionalen Weltwirtschaft ${ }^{1}$. Notwendigkeit und potentielle Vorteile der Wirtschaftsintegration zwischen Entwicklungsländern sind seit dem „Allgemeinen Prinzip 10" der UNCTAD I (1964) in zahlreichen UN- und UNCTAD-Resolutionen weltweit anerkannt und ergeben sich im wirtschaftlichen Bereich vor allem aus der Tatsache, daß 1967 rund 80 Prozent der Entwicklungsländer weniger als 15 Mill. Einwohner und 53 Entwickungsländer weniger als fünf Mill. Einwohner und damit angesichts der geringen Kaufkraft der Bevölkerung viel zu enge Binnenmärkte für eine rentable Industrialisierung hatten; in dieser Situation ermöglicht die Wirtschaftsintegration ein Ausnutzen der Vorteile der Großraumwirtschaft, vermindert die Gefahr einer Vergeudung der knappen Investitionsmittel infolge unrentabler Parallelinvestitionen in jedem einzelnen Land und kann die notwendige Verbesserung der Außenhandelsposition (z. B. der terms of trade) sowie des seit 1950 rückläufigen Welthandelsanteils der Entwicklungsländer (1950 31,6 Prozent, 1971 17,6 Prozent) erleichtern².

Die jeweiligen Integrationsmethoden der Entwicklungsländer waren in der Praxis zwar sehr unterschiedlich: Wirtschafts- und Währungsunionen, Gemeinsame Märkte, Zollunionen, Freihandelszonen, sektorell begrenzte Präferenzzonen oder andere Projekt- und Kooperationsabkommen, die mitunter wie z. B. die Cocoa Producers' Alliance (Brasilien, Elfenbeinküste, Ghana, Kamerun, Nigeria und Togo) auch eine interkontinentale Wirtschaftskooperation anstreben und hinsichtlich ihrer wirtschaftlichen Bedeutung, ihrer Mitgliederzahl und des erreichten "Integrationsgrads“ erhebliche Unterschiede aufweisen. Infolge der zahlreichen Integrationshindernisse infrastruktureller, wirtschaftlicher, politischer, sozialer, sprachlicher und psychologischer Art und der nur bei wenigen dieser Abkommen (Andenmarkt, CACM, UDEAC, EAC, OPEC) genügend starken, politischen Integrationsbereitschaft haben die meisten dieser Integrationsabkommen, besonders in Asien und Westafrika, ihre wirtschaftlichen Integrationsziele bislang jedoch nicht oder nur teilweise er-

\footnotetext{
Abkürzungen: $\mathrm{CACM}=$ Central American Common Market; CARIFTA = Caribbean Free Trade Association; $\mathrm{EAC}=$ East African Community; LAFTA = Latin American Free Trade Area; OCAMM $=$ African, Malagasy and Mauritian Common Organization; OERS = Organisation des Etats riverains du tleuve Sénégal; UDE = Union Douanière Equatoriale; UDEAC = Union Douanière et Economique de l'Atrique Centrale.

$1 \mathrm{Zum}$ neuesten Integrationsstand vgl. Survey of Economic Conditions in Africa 1971, ECA 1972, S. 196 ff.; Y. Lim, ECAFE's Trade Expansion Proposal for Asia, Journal of Common Market Studies, Dez. 1972 , S. 103 ff.; Le Mouvement d'Intégration en Amérique Latine, in: Problèmes Economiques, 10.1. 1973 , S. 16-25.

2 Ausführlich zu den ökonomischen Vorteilen: Eckenstein, Die wirtschaftliche Zusammenarbeit und regionale Integration zwischen Entwicklungsländern, Außenwirtschaft 1972, S. 28 ff.
} 
reicht $^{3}$. Auffällig - und den Ansatzpunkt für die folgenden völkerrechtlichen Ausführungen bildend - ist hierbei die in den zahlreichen UNCTAD-Expertenanalysen $^{4}$ zur Integrationspraxis der Entwicklungsländer festgestellte Ahnlichkeit völkerrechtlich relevanter Integrationshindernisse: der bislang geringe Erfolg bei der Verwirklichung einer regionalen Investitionspolitik, die geringe währungspolitische Kooperation, unzureichende institutionelle Regelungen und Gemeinschaftskompetenzen sowie als schwierigstes Hindernis das Problem einer gerechten Verteilung der Kosten und Vorteile der Wirtschaftsintegration. Die Krisen im CACM (Fußballkrieg 1969 usw.), in der UDEAC (Austritt von Tschad und Zentralafrikanischer Republik 1968) und in der EAC (Wiedereinführung von internen Handelsschranken 1971) wurden sämtlich durch die "Gewinnkonzentration“ in entwickelteren Mitgliedstaaten mitverursacht und führten dann zum Austritt einzelner Mitgliedstaaten, zu einem mühevollen Umstrukturierungsprozeß (EAC, CACM) oder zu einseitigen, desintegrierenden Maßnahmen der Mitgliedstaaten (Aufhebung des Freihandels seitens Honduras im CACM oder der Konvertibilität in der EAC). Auch bei sektorell begrenzten Kooperationsabkommen traten dieselben Schwierigkeiten auf (z. B. 1970 der Rücktritt Senegals vom OCAMM-Zuckerabkommen), zumal hier der geringeren Kohärenz eine größere Bereitschaft zur Bevorzugung der nationalen gegenüber den regionalen Interessen gegenübersteht. Als Voraussetzung für eine gerechte Verteilung der Integrationsvorteile nennen die UNCTAD-Expertenanalysen daher: handelspolitische Präferenzmaßnahmen zugunsten der weniger entwickelten Partnerstaaten (z. B. bei der Handelsliberalisierung, der Übernahme des GZT, den Ursprungsregeln usw.), wie sie z. B. innerhalb der Anden-Gruppe zugunsten von Bolivien und Ecuador, innerhalb der CARIFTA für acht oder zwölf Mitgliedstaaten oder innerhalb der LAFTA zugunsten von vier Mitgliedern vorgesehen sind; Zahlungsbilanzhilfen der Länder mit einer aktiven Handelsbilanz zugunsten der weniger entwickelten Länder mit chronischem Defizit im innerregionalen Handel, wie sie z. B. in dem von den LAFTA-Staaten und der Dominikanischen Republik im September 1969 unterzeichneten „Santo-DomingoAbkommen" vereinbart sind; gemeinschaftliche Mechanismen zum Ausgleich integrationsbedingter Steuereinnahmeverluste, wie z. B. das "transfer-tax“-System in der EAC, die "taxe unique“ in der UDEAC oder der Solidaritätsfonds in der UDE; Steuerharmonisierung und Bevorzugung der weniger entwickelten Mitgliedstaaten bei steuerlichen Industrialisierungsanreizen, was z. B. in einem 1969 in Kraft getretenen Abkommen der CACM-Staaten zugunsten von Honduras vorgesehen ist; regionale Entwicklungsplanung zur Koordinierung und Lenkung der industriellen Entwicklung (z. B. „Integrationsindustrien“ im CACM, „Komplementaritätsabkommen" in der LAFTA, der gemeinschaftliche Investtiionskodex in der UDEAC, regionale Entwicklungsbanken in der EAC und CARIFTA) und der regionalen Infrastruktur (z. B. gemeinsame Infrastrukturprojekte in der OERS, gemeinsame Dienstleistungsorganisationen in der EAC); Harmonisierung der Agrarpolitiken (vgl. das im Dezember 1971 von den Ländern der Anden-Gruppe vereinbarte landwirtschaftliche Integrationsprogramm oder die zahlreichen gemeinsamen Ver-

\footnotetext{
3 Nach Wionczek (Hg.), Economic Cooperation in Latin America, Africa and Asia, 1969, S. V, funktionierten 1968 nur 9 wirtschaftliche Regionalabkommen mit einer Mitgliederzahl von insgesamt 46 Entwicklungsländern. Ahnlich der OECD-Expertenbericht: Economic Integration Among Developing Countries, OECD 1969, S. 132: "In conclusion it can be said that even those we have examined, which are the most advanced of the integration schemes in the developing world, are still far from reaching their objectives».

4 Vgl. zuletzt: TD/B/333, 1970; TD/B/322/Rev. 1, 1971; TD/B/374, 1971; TD/110, 1972; TD/99, Ziff. 338, $1972 ; \mathrm{TD} / \mathrm{B} / 413,1972$.
} 
kaufsorganisationen wie die Afro-Madagassische Kaffeeorganisation und die Asiatische Kokosnußgemeinschaft); subregionale Finanzierungsinstitutionen zur Förderung der Wirtschaftsintegration und zur Mobilisierung externer Finanzhilfe; externe handelspolitisché, finanzielle und technische Unterstützung seitens der Industrieländer und internationalen Organisationen.

$\mathrm{Da}$ diese Integrationsmaßnahmen regelmäßig nur mit Hilfe des Rechts durchführbar sind und in integrationsrechtlichen Vereinbarungen vorausgeplant sowie gegebenenfalls dem Art. XXIV GATT angepaßt werden müssen, stellt sich die Frage, welche Rechts- und Organisationsformen aufgrund der bisherigen Integrationserfahrungen als am vorteilhaftesten für die verschiedenen Arten regionaler Wirtschaftsintegration zwischen Entwicklungsländern anzusehen sind.

\section{Eine völkerrechtliche Integrationstheorie?}

Besonders die OKonomen ${ }^{5}$ betonen, daß regionale Wirtschaftsgemeinschaften zugleich Rechtsgemeinschaften sein müssen: umfassende Wirtschaftskooperation muß sich funktionsnotwendig des Rechts als Instrument der Planung sowie der einheitlichen, nichtdiskriminierenden Gestaltung der Wirtschaftsintegration bedienen, und die wirtschaftliche setzt daher eine rechtliche Integration voraus. Im Gegensatz zu den zahlreichen, die Integrationspraxis sichtlich beeinflussenden ökonomischen und politologischen Integrationstheorien hat sich die Völkerrechtswissenschaft bislang jedoch kaum mit der Frage nach einer allgemeinen Theorie des Rechts der Wirtschaftsintegration befaßt ${ }^{6}$.

Im wirtschaftswissenschaftlichen Bereich hat die in den 50er Jahren im Hinblick auf die europäische Integration entwickelte "klassische Theorie“ der Zollunion (J. Viner, J. E. Meade, R. G. Lipsey) vorwiegend nur die (statisch) unmittelbaren Auswirkungen der Zollunion auf Produktion, Nachfrage, Handelsströme, terms of trade und auf die Wohlstandssteigerung im Vergleich zu einem hypothetischen Freihandels- oder „second-best“-Optimum untersucht und die „dynamischen“ Integrationsvorteile (z. B. Produktivitätssteigerung infolge größerer Betriebseinheiten und größeren Wettbewerbs) meist unterschätzt oder unterschiedlich bewertet ${ }^{7}$. Während der 60er Jahre wurde die ökonomische Integrationstheorie wesentlich weiterentwickelt ${ }^{8}$, wobei u. a. H. Bourguinat ${ }^{9}$ die Inadäquanz der klassischen Zollunion-Theorie für die Wirtschaftsintegration zwischen Entwicklungsländern nachgewiesen hat und in Übereinstimmung mit den UNCTAD-Expertenanalysen betont, daß entgegen der Forderung des Art. XXIV GATT nach Null-Zolltarifen die potentiellen Integrationsvorteile zwischen Entwicklungsländern am besten durch zunächst partielle oder sektorell begrenzte Präferenzzonen ausgenutzt werden können und letztere zur Verwirklichung einer echten Solidaritäts- und Risikogemeinschaft durch gemeinsame oder zumindest koordinierte Infrastruktur-, Industrialisierungs- und Entwicklungspolitiken ergänzt werden müssen.

Bei den Bemühungen amerikanischer Politologen wie E. Haas, P. Schmitter, K. W. Deutsch, J. Nye und A. Etzioni um ein allgemeines, theoretisches Modell für die

\footnotetext{
5 Vgl. z. B. Röpke, Economic order and international law, RdC 1954 II, S. 211, 218.

6 Eine Ausnahme ist die vorwiegend die lateinamerikanische Integrationspraxis untersuchende Arbeit von F. Villagran, Teoria General Del Derecho De Integración Económia, Ensayo de Sistematización, Costa Rica 1969. Um eine rechtsvergleichende Systematisierung bemühen sich: Dag Hammarskjöld Seminar, Legal Aspects of Economic Integration, Uppsala 1970; Legal Aspects of Economic Integration, R. d. C., Colloquium 1971, Leiden 1973. Speziell zum Europäischen Gemeinschaftsrecht vgl.: P. Pescatore, Le Droit de l'Intégration, 1972.

$7 \mathrm{Vgl}$. den berühmten Aufsatz von R. G. Lipsey, The Theory of Customs Unions: A General Survey, in: Economic Journal, LXX Sept. 1960, S. 496-513.

$8 \mathrm{Vgl}$. Krauss, Customs Union Theory: Ten Years Later, Journal of World Trade Law 3/1972, S. 284 ff.

$9 \mathrm{H}$. Bourguinat, Les marchés communs des pays en voie de développement, 1968, S. $75 \mathrm{ff}$.
} 
Strukturen, Entwicklungsstufen und Dynamik der Integration von Nationalstaaten zu größeren politischen Einheiten stehen sich die Konstitutionalismustheorie und die Funktionalismustheorie gegenüber ${ }^{10}$. Während nach Ansicht der Konstitutionalisten die staatlichen Ordnungs- und Wertprinzipien sich auch in der politischen Regionalintegration als Strukturprinzipien durchsetzen müssen und die Vergemeinschaftung daher eine mit den Verfassungs- und Regierungssystemen der Mitgliedstaaten homogene, bundesstaatliche oder ähnliche Überstaatsbildung anstrebt („function follows form"), empfehlen die Funktionalisten die Übertragung der auf einzelstaatlicher Ebene nicht mehr zu erfüllenden Aufgaben zur gemeinschaftlichen Wahrnehmung in supranationalen Entscheidungsorganen, wobei die größere Sachgerechtigkeit und Rationalität funktioneller Gemeinschaftsregelungen die Vergemeinschaftung zusätzlicher, interdependenter Sachbereiche begünstigt („spill over“). Hinsichtlich der Form des Funktionsverbandes wird der Integrationsprozeß von den Funktionalisten als prinzipiell offenes System verstanden, wobei aber jedem soziopolitischen Verband als einem „social system of behaviour" für die Systemerhaltung funktionsnotwendige Strukturen und eine gewisse Eigendynamik immanent sind („form follows function "). Im Hinblick auf die Frage nach einer völkerrechtlichen Integrationstheorie ist jedoch das Eingeständnis der Politologen wichtig, daß die großen Unterschiede $z$ wischen den regionalen Wirtschaftsgruppierungen und die noch unzureichende Bestandsaufnahme bislang eine allgemeingültige Integrationstheorie verhinderten: "regional integration theory has failed to provide the required guidance to achieve the goal to integration" 11 .

Hinsichtlich der Möglichkeit und Voraussetzungen einer völkerrechtlichen Integrationstheorie ist es angesichts der Interdependenz zwischen den wirtschaftlichen, politischen und rechtlichen Integrationsebenen und des instrumentalen Charakters des Rechts zweifelhaft, ob das weitgehend von der sozioökonomischen und politischen Situation vorgeformte Integrationsrecht ohne ausreichende politologische Vorarbeiten allein durch eine gemeinschaftsrechtliche „Integrationstheorie“ erklärt werden kann. Die Abhängigkeit rechtspolitischer Stellungnahmen zu zahlreichen Bereichen des Integrationsrechts von vorherigen politologischen Untersuchungen hat H. P. Ipsen jüngst mit Recht betont ${ }^{12}$. Weiter ist es eine durch die Integrationspraxis der Entwicklungsländer bestätigte Tatsache, daß ebenso wie die ökonomischen auch die rechtlichen Integrationsprobleme je nach Entwicklungsstand und regionalen Besonderheiten unterschiedlich sein können: so kann das in der EWG bewährte, rechtliche und institutionelle Integrationsmodell nicht auf Entwicklungsländer übertragen werden, wenn letztere z. B. sich ein Initiativ- und Exekutivorgan wie die EG-Kommission mit über 5000 Planstellen und einem Budget in Höhe von fast $4 \mathrm{Mrd}$. RE (Haushaltsjahr 1972) mangels finanzieller und technischer Mittel weder leisten können noch angesichts der geringen Bedeutung des regionalen Präferenzzonenhandels für den gesamten Außenhandel dieser Entwicklungsländer (durchschnittlicher Anteil 1969 8,1 Prozent) leisten wollen. Während innerhalb der EWG die Rechtsstaats- und Demokratieprinzipien der Mitgliedstaaten die Errichtung gerichtlicher und parlamentarischer Gemeinschaftsorgane notwendig machten, konnten in den lateinamerikanischen Integrationsabkommen auf derartige Gemeinschaftsorgane durchweg verzichtet werden. Angesichts ihrer beson-

10 Vgl. z. B.: G. Zellentin, Intersystemare Beziehungen in Europa, 1970, S. $172 \mathrm{ff}$.

11 R. D. Kaiser, Toward the Copernican Phase of Regional Integration Theorie, Journal of Common Market Studies, März 1972, S. 207 ff., 232.

12 Ipsen, Europäisches Gemeinschaftsrecht, 1972, S. 17 f. 
deren ökonomischen Integrationsprobleme haben die Entwicklungsländer mit Hilfe der UNCTAD und der regionalen UN-Wirtschaftskommissionen eine auch in der GATT-Praxis zu Art. XXIV GATT bestätigte, weltweite Anerkennung ihrer Forderung erreicht, daß wirtschaftliche Integrationsabkommen zwischen Entwicklungsländern sich über Art. I, XXIV GATT hinaus aller als entwicklungspolitisch notwendig erachteten Rechtsstrukturen bedienen können ${ }^{13}$. Die Integrationsabkommen zwischen Entwicklungsländern sind schließlich auch untereinander infolge von Unterschieden bei den Integrationszielen, bei der wirtschaftlichen Komplementarität, politischen Homogenität und rechtlichen Traditionen sehr verschieden. Trotz dieser regionalen Besonderheiten und unterschiedlichen Integrationstechniken zeigen sich bei einer rechtsvergleichenden Untersuchung der Wirtschaftsintegrationsabkommen neben dem gemeinsamen Ziel der Handelsliberalisierung und Entwicklungskooperation zahlreiche weitere rechtliche, institutionelle und strukturelle Gemeinsamkeiten sowie das Integrationsrecht verformende wirtschaftliche, politische und rechtliche Sachzwänge. Grundlagen der rechtlichen Integration sind der völkerrechtliche Integrationsvertrag und das darauf beruhende Sekundärrecht, aber auch die Harmonisierung und Angleichung der nationalen Rechtsordnungen und privatrechtliche Lösungen (internationales Privatrecht, transnationales Handelsrecht). Die größere Homogenität, Interdependenz und Solidarität zwischen regional benachbarten Partnerländern ermöglichen dabei häufig weitergehende Lösungen als auf weltweiter Ebene, und die nicht nur für das Recht der Europäischen Gemeinschaften, sondern ebenso für das wirtschaftliche Integrationsrecht der LAFTA, des CACM, des Andenmarkts oder der UDEAC üblich gewordene Bezeichnung als "Gemeinschaftsrecht" weist dabei auf die zunehmende Tendenz hin, dem regionalen Wirtschaftsgemeinschaftsrecht eine besondere „Rechtsqualität“ zuzuschreiben. Die wirtschaftliche Integrationsdynamik und Stufenplanung erfordern flexible Anpassungs- und Schutzmechanismen im Integrationsrecht und regelmäßig eine die wirtschaftliche Entwicklung vorauskonzipierende Wirtschafts- und Planungsverfassung. Beim Integrationsvertrag wird es sich daher meist um einen auf exekutiven, normierenden und rechtsprechenden Vollzug durch die Mitgliedstaaten und Gemeinschaftsorgane angelegten Rahmenvertrag („traité-cadre“) handeln. Das Gemeinschaftsrecht muß zur Sicherung der Marktgleichheit seine einheitliche Geltung in den Mitgliedstaaten anstreben und muß mit Hilfe des aus den integrationsrechtlich vereinbarten Gemeinschaftsaufgaben zu entnehmenden „Prinzip der Sicherung der Funktionsfähigkeit“ derart interpretiert und qualifiziert werden, daß der bezweckte Erfolg im höchstmöglichen Grade gewährleistet ist. Weitere rechtliche Parallelen $\mathrm{zwischen}$ den verschiedenen Integrationsabkommen ergeben sich z. B. im Hinblick auf Art. XXIV GATT sowie aus der Ähnlichkeit der zu regelnden Sachbereiche und wirtschaftlichen Funktionszwänge wie z. B. des aus umfassender Handelsliberalisierung oft resultierenden Zwangs zur Währungskooperation (z. B. multilateraler Zahlungsausgleich ${ }^{14}$ ) oder aus mangelnder Währungskooperation resultierenden Gefährdung gemeinsamer Agrarmarktordnungen („spill back“).

Aufgabe einer völkerrechtlichen Integrationstheorie müßte die Systematisierung und Erklärung der rechtlichen Parallelen und Unterschiede im jeweiligen regionalen Integrationsrecht der Wirtschaftsgemeinschaften (zwischen Entwicklungsländern) und eine auf dem empirischen Vergleich von Integrationszielen und Inte-

13 Vgl. Petersmann, Das neue Recht des Nord-Süd-Handels, ZaöRV 1972, S. 339 ff.: 377 ff.; G. de Lacharrière, La Nouvelle Division Internationale du Travail, 1969, S. $67 \mathrm{f}$.

14 Vgl.: Payments arrangements among the developing countries for trade expansion, TD/B/80, 1966. 
grationserfolgen beruhende Bewertung der jeweiligen Formen und Methoden des Integrationsrechts sein. Die praktische Bedeutung einer völkerrechtlichen, empirisch abgesicherten Integrationstheorie stünde außer Zweifel: ebenso wie die erwähnten, ökonomischen Expertenanalysen nachweislich die Integrationspraxis beeinflussen und $\mathrm{zu}$ immer ähnlicheren Integrationstechniken der Entwicklungsländer führen (z. B. regionale Entwicklungsbanken, multinationale Investitionskodices, Vorzugsmaßnahmen für weniger entwickelte Partnerländer), könnte auch die Ermittlung und unter Umständen modifizierte Übernahmen erfolgreicher rechtlicher Integrationsmethoden (z. B. das in der EWG erfolgreich praktizierte und seit 1969 auch im Rahmen des Andenmarktes nachgeahmte Vorschlags- und Initiativrecht eines eigenständigen Gemeinschaftsorgans) zum größeren Erfolg wirtschaftlicher Integrationsabkommen beitragen. Eine Systematisierung des Integrationsrechts ist dabei nach zahlreichen Gesichtspunkten möglich ${ }^{15}$. Der folgende Abschnitt beschränkt sich auf rechtsvergleichende Anmerkungen zu drei zentralen Problemen jeden Wirtschaftsintegrationsrechts (Planungscharakter, Verhältnis zum Landesrecht, institutionelle Regelungen), ohne dabei die von wesentlich umfassenderen, empirischen Untersuchungen abhängige Problematik einer allgemeinen völkerrechtlichen Integrationstheorie weiter zu verfolgen.

\section{Rechtsvergleichende Aspekte des Wirtschaftsintegrationsrechts der Entwicklungsländer}

\subsection{Die Integrationsabkommen als Planungsverfassungen}

Die wirtschaftlich, sozial und politisch komplexen, sich dynamisch weiterentwikkelnden Probleme regionaler Wirtschaftsintegration und der besonders in Lateinamerika verbreitete, einseitig nationale Souveränitätsmythos machen in der Anfangsphase die Beschränkung auf einen lediglich die Integrationsziele sowie die rechtliche und institutionelle Grundordnung regelnden Rahmenvertrag („traitécadre") erforderlich, der seine rechtliche Ergänzung und Weiterentwicklung voraussetzt oder doch zumindest zuläßt. Da die Integrationsverträge regelmäßig gemeinsame Exekutiv- und Rechtssetzungsorgane errichten, deren abgeleitete Rechtsakte dem primären Integrationsrecht entsprechen müssen, handelt es sich zugleich um über die Kategorie des „Gesetzesvertrags“ („traité-loi“) hinausgehende „Verfassungsverträge“ („traité-constitution“). Insofern die Integrationsabkommen darüber hinaus Ziele, Mittel, Prioritäten, Stufenfolge sowie Funktions- und Organisationsweise der Wirtschaftsintegration vorausplanen und eine der Anpassung und Fortschreibung der Planung korrespondierende Änderung des Planungsrechts voraussetzen, kann man diese Verträge auch als „Planungsverfassungen“ qualifizieren $^{16}$.

15 Vgl. z. B.: H. R. Krämer, Formen und Methoden der internationalen wirtschaftlichen Integration, Versuch einer Systematik, 1969, oder die von Pescatore (Das Zusammenwirken der Gemeinschaftsrechtsordnung mit den nationalen Rechtsordnungen, EuR 1978, S. 307) vorgenommene Einteilung des Integrationsrechts in Primär-, Sekundär-, Tertiär- und Quartärschichten, je nachdem ob die integrationsrechtlichen Zielsetzungen durch gemeinschaftliche Rechtssetzungs-, Rechtsanwendungs- und Vollzugskompetenzen ergänzt werden.

$16 \mathrm{Zu}$ den Kategorien "traité-contrat", "traité-loi“, "traité-cadre“ und "traité-constitution“ vgl. z. B. C. F. Ophüls, Die Europäischen Gemeinschaftsverträge als Planungsverfassungen, in: J. H. Kaiser (Hrsg.) Planung I, 1965, S. $229 \mathrm{ff}$. 
Die Charakterisierung der Integrationsverträge als „Planungsverfassungen“ wird durch die Flexibilität und die infolge des Planungscharakters oft nur "relative Normativität" des Integrationsrechts verdeutlicht:

a) Die rechtliche Flexibilität zeigt sich einerseits in den Vertragsänderungsverfahren und umfangreichen Schutz- und Ausnahmeklauseln. Neben der in den Integrationsverträgen regelmäßig vorgesehenen Änderungsmöglichkeiten durch einen „acte contraire" 17 hat vor allem die LAFTA-Praxis zahlreiche Änderungen und Ergänzungen des Integrationsvertrages durch Resolutionen der Gemeinschaftsorgane oder durch Regierungsabkommen verwirklicht. Die Schutzklauseln sind wegen des unterschiedlichen Entwicklungsstands der Partnerländer besonders wichtig und lassen u. a. zum Schutz der ,infant industries“, der nationalen Agrarproduktion, der Sicherheit und öffentlichen Ordnung, bei Zahlungsbilanzdefiziten oder aus fiskalischen Gründen unter jeweils unterschiedlichen Voraussetzungen (temporäre, nichtdiskriminierende Anwendung, vorherige Konsultation usw.) die einseitige Einführung von Zöllen und Kontingenten zu. Hinsichtlich der afrikanischen Integrationsabkommen sind besonders zu erwähnen: in der UDEAC das "taxe-unique“System (Art. 59 ff.), die Zulässigkeit nationaler, entwicklungspolitisch bedingter Einfuhrkontingente gegenüber Drittstaaten (Art. 40), die klassische Schutzklausel für den Fall grundlegender Störungen in einem Wirtschaftsbereich (Art. 41) und in einem weiteren Sinne auch der Gemeinsame Solidaritätsfonds (Art. 38); in der EAC das auf 15 Jahre befristete „transfer tax“-System (Art. 20), die Zulässigkeit gewisser mengenmäßiger Beschränkungen für den Agrarhandel innerhalb der EAC (Art. 13), die einseitigen Handelsbeschränkungen aus Gründen der Sicherheit, der öffentlichen Ordnung, beim Abweichen eines anderen Partnerstaates von der gemeinsamen Verbrauchssteuer oder wegen Zahlungsbilanzschwierigkeiten (Art. 12), und in einem weiteren Sinne auch die Errichtung der Ostafrikanischen Entwicklungsbank; in der UDEAO die Zulässigkeit von Fiskal- und Schutzzöllen und in gewissen Fällen (wirtschaftliches Ungleichgewicht, gewisse Fertigwaren) auch von Kontingenten (Art. 6,7) gegenüber Einfuhren aus anderen Partnerstaaten; in dem Zollunion-Abkommen mit Südafrika die Zulässigkeit interner, auf acht Jahre befristeter Zölle seitens Betschuana, Lesotho und Swaziland zum Schutz ihrer „infant industries" gegenüber konkurrierenden Erzeugnissen aus dem Zollgebiet (Art. 6) und ihr Vetorecht gegenüber einer Änderung der diesbezüglichen Außenzölle (Art. 7).

b) Kennzeichnend ist weiter die besonders in Lateinamerika dominierende Rolle des sekundären Integrationsrechts und ergänzender Regierungsabkommen, wobei die Integrationspraxis häufig weit über die ursprünglichen Rahmenregelungen hinausführt ${ }^{18}$ und dem Integrationsrecht Züge einer "living constitution" verleiht. Der zentralamerikanischen Wirtschaftsintegration liegen z. B. über 38 multilaterale Verträge zugrunde ${ }^{19}$. Der LAFTA-Vertrag wird durch zahlreiche nachträgliche Protokolle (z. B. die am 12. 12. 1966 unterzeichneten Protokolle über die Freizügigkeit, die Beilegung von Streitigkeiten und über die Errichtung des

\footnotetext{
17 Vgl. z. B. Art. 94/1 EACV: "This Treaty may be modified at any time by agreement of all the Partner States". Art. 60 LAFTAV: "The Contracting Parties may present amendments to the present Treaty which shall be set out in protocols that shall enter into force upon their ratification by all the Contracting Parties and after the corresponding instruments have been deposited“. - Die Texte the Contracting Parties and after the corresponding instruments have been deposited ". - Die Texte
der Integrationsverträge finden sich u. a. bei Wionczek (Anm. 3); L. B. Sohn (Hg.), Basic Documents of African Regional Organizations, Bd. I-IV, 1971, 1972, 1973; sowie in des International Legal Materials.

18 Vgl. F. Orrego Vicuña, RdC, Colloquium 1971 (Anm. 6), S. 165, 402 f., 506 f.

19 Derecho de la Integración latino-americana, 1969, S. $966 \mathrm{ff}$.
} 
Rats der Außenminister als LAFTA-Organ), durch andere internationale Abkommen (z. B. das im September 1965 gebilligte Abkommen der Zentralbanken über den Ausgleich von Zahlungen und gegenseitigen Krediten), durch mehrere hundert Resolutionen der LAFTA-Organe (vgl. z. B. Resolution 222, Nr. 7 zur Vereinbarkeit des Cartagena-Abkommens über die Gründung des Andenmarkts mit der LAFTA: „Tariff reductions stipulated in a subregional agreement shall not extend to contracting parties which do not participate in it, nor will they create for them special obligations") und durch auf Regierungskonferenzen gefaßte Beschlüsse (z. B. die Erklärung der Amerikanischen Präsidenten von Punta del Este, 1967) ergänzt. Dieses nachträgliche Integrationsrecht hat teils zu einer weit über den anfangs oft nur handelspolitischen Bereich hinausgehenden Erweiterung der Integrationsmaterie geführt: so sieht die am 8. 12. 1964 von der LAFTA-Konferenz gebilligte Resolution 100 (IV) z. B. eine begrenzte Ausarbeitung einheitlicher Konjunktur-, Transport-, Währungs-, Finanz- und Zahlungspolitiken vor, und die am 2. 9. gefaßten Resolutionen 202 und 203 des LAFTA-Ministerrats über die Vereinbarkeit subregionaler Abkommen, insbesondere des Cartagena-Abkommens, mit der LAFTA stellen ebenfalls eine materiell-rechtliche Erweiterung des LAFTARechts dar (z. B. der Meistbegünstigungspflichten in Art. 18-20).

Das „Folgerecht“ hat zugleich zu Änderungen des ursprünglichen Integrationsabkommens geführt: so ermächtigt z. B. die LAFTA-Resolution 209 die relativ weniger entwickelten LAFTA-Staaten $\mathrm{zu}$ einer von Art. 4/6 abweichenden, langsameren Handelsliberalisierung, und das Außenministertreffen von 1965 sowie die Erklärung der Amerikanischen Präsidenten von 1967 sehen entgegen Art. 4 ff. eine automatische Handelsliberalisierung im Rahmen der LAFTA vor. Das Prinzip des Vorrangs des Primärrechts hat sich in Lateinamerika ebenso wie in der EWG als ein nur schwaches Hindernis gegenüber einer Vertragsänderung durch nachträgliche Beschlüsse und „accords en forme simplifiée“ der Regierungsvertreter erwiesen. Die Kompetenzen und Rechtsakte der Gemeinschaftsorgane (Resolutionen, Ubereinkommen und Entscheidungen in der LAFTA, Entscheidungen und Richtlinien im Anden-Markt, Resolutionen und Empfehlungen im CACM usw.) sind darüber hinaus in den lateinamerikanischen Integrationsabkommen meist nur vage und ohne präzise Systematik geregelt. Angesichts des Fehlens gemeinschaftlicher Rechtssprechungsorgane in den lateinamerikanischen Integrationssystemen ist eine übereinstimmende, von allen Vertragsparteien getragene Integrationspraxis daher nahezu "omnipotent": „in many cases the process of integration develops itself through channels of uncertain legality, does not guarantee to the necessary extent legal stability, accepts openly illegal violations and, finally, rests more upon the benefits of political negotiations than those of the rule of law"... "provided there exists a consensus between governments, decisions of any kind, nature or extend can be enacted even if they are manifestly contrary to justice, incur an abuse or 'détournement' of power or directly violate the legality of the regional order" 20.

Die politische, soziale und administrative Instabilität innerhalb der lateinamerikanischen Länder, die teils politisch-ideologischen Gegensätzen sowie das wirtschaftliche Ungleichgewicht zwischen diesen Ländern bewirken zwangsläufig auch eine gewisse Instabilität auf der gemeinschaftlichen Integrationsebene. Nach lateinamerikanischer Ansicht ist eine "hétérodoxie juridique . . . tout à fait adaptée au

20 F. Orrego Vicuña (Anm. 18), S. 103, 104. 
besoin de l'intégraton et du dévelopment de l'Amérique latine, parce qu'au contraire dans certains cas, la seule façon de dépasser une situation de conflit serait la rupture du processus d'intégration, et parfois une rupture de la légalité est préférable à la rupture du processus d'intégration. Je crois qu'il est très important de comprendre qu'en Amérique latine parfois l'illégalité est une source de légalité dans le droit interne et surtout dans le droit international. Il faut faire des actes illégaux pour réadapter dans certains circonstances les projets d'intégration à la réalité politique des pays participanis"21. Diese Abhängigkeit der „normati-

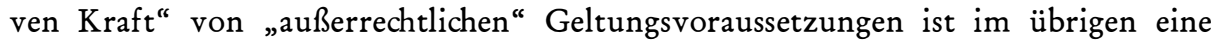
auch in der EWG (vgl. den „Luxemburger Kompromiß“ von 1966, die nachträgliche Billigung der einseitigen Maßnahmen Frankreichs während der Währungskrise 1968 seitens der EWG) und in anderen Bereichen des internationalen Wirtschaftsrechts sichtbare Realität (z. B. beim nach der IMF-Satzung nicht genehmigungsfähigen „Floaten“ der wichtigsten europäischen Währungen 1972/73), zumal das wirtschaftliche Planungsrecht nicht absolut gelten, sondern das Planziel verwirklichen soll und daher eine laufende Planfortschreibung voraussetzt.

c) Während die Regierungen der Vertragsparteien somit sehr weit „von außen “ in das Integrationsrecht hineinwirken und es verändern können, sind die direkten Mitwirkungsmöglichkeiten für die nationalen Parlamente und den privaten Wirtschaftssektor durchweg gering. Parlamentarische Integrationsorgane sind in keinem der Abkommen vereinbart: das 1964 gegründete „Lateinamerikanische Parlament“ ist nicht mit dem wirtschaftlichen Integrationsprozeß verbunden, und die Mitglieder der Ostafrikanischen Gesetzgebenden Versammlung brauchen gemäß Art. 57 EACV keine Parlamentsmitglieder zu sein. Im Falle der LAFTA wird die parlamentarische Billigung des LAFTA-Vertrages als generelle Ermächtigung der Exekutive zur Durchführung aller aus dem Integrationsvertrag resultierenden Verpflichtungen interpretiert ${ }^{22}$. In der LAFTA wurde durch die Resolutionen 74 und 75 des Exekutivausschusses zwar eine Beteiligung der Arbeitnehmer und Geschäftsleute im Rahmen von Beratenden Ausschüssen für Wirtschafts- bzw. Arbeitsangelegenheiten vorgesehen, und auch das Cartagena-Abkommen sieht einen beratenden Wirtschafts- und Sozialausschuß für die Beteiligung der Arbeitnehmer und Arbeitgeber vor. Im Bereich der LAFTA wird die Beteiligung des privaten Wirtschaftssektors dennoch als völlig unzureichend angesehen, wichtige politische Entscheidungen fallen ohne wirksame Konsultation der Arbeitnehmer- und Arbeitgeberkreise, und angesichts des Fehlens gemeinschaftlicher Rechtsprechungsorgane in den wirtschaftlichen Integrationssystemen Lateinamerikas ist der gerichtliche und administrative Rechtsschutz des Privatsektors gegenüber der Exekutive im Widerspruch $\mathrm{zu}$ seiner dynamischen Funktion im Integrationsprozeß gering.

\subsection{Integrationsrecht und Landesrecht}

Die mit regionaler Wirtschaftsintegration verbundene rechtliche Integration führt zwangsläufig zu einer Überlagerung und Änderung des nationalen Wirtschaftsrechts durch das wirtschaftliche Integrationsrecht. Die jeder regionalen Wirtschaftsintegration eigenen Prinzipien der Nichtdiskriminierung und Marktgleich-

21 F. Peña, RdC, Coloquium 1971, S. 439 f

22 F. Orrego Vicuña (Anm. 18), S. 144 f., 146. 
heit erfordern dabei regelmäßig eine einheitliche, gleichzeitige Anwendung des Integrationsrechts in allen Mitgliedstaaten. Eine rechtliche Regelung der Symbiose $\mathrm{zwischen}$ nationalem und regionalem Wirtschaftsrecht ist daher funktionsnotwendig.

Während in den Europäischen Gemeinschaften der Vorrang (vgl. Rechtssache 6/64), die unmittelbare Wirkung (vgl. Rechtssache 26/62), die einheitliche Anwendung des Gemeinschaftsrechts (vgl. Art. 169 f., 173, 175, 177 usw. EWGV), der den gemeinschaftlichen Rechtsetzungskompetenzen meist korrespondierende Verlust nationaler "Parallelkompetenzen" (Rechtssachen 26/62, 22/70) oder der lange Zeit umstrittene Umfang der gemeinschaftlichen „treaty making power" (Rechtssache 22/70) erst durch die kühne „judicial legislation“ des Europäischen Gerichtshofs gesichert und rechtsverbindlich entschieden wurden, haben das Fehlen gemeinschaftlicher Rechtsprechungsorgane und das nationale Souveränitätsdenken besonders bei den lateinamerikanischen Integrationsabkommen eine klare Regelung der Rechtsbeziehungen zwischen Integrationsrecht und Landesrecht verhindert. Das regionale Integrationsrecht der Entwicklungsländer weist zwar gegenüber dem allgemeinen Völkerrecht besondere Aspekte auf (z. B. vereinfachte Ratifizierungs- bzw. „Transformations"-Verfahren, größere Zahl unmittelbar anwendbarer Normen, direkte Einbeziehung der Unternehmen, Sozialpartner und Individuen, Mehrheitsbeschlüsse). Die Anwendung der traditionellen Völkerrechtsregeln für die Beziehungen zwischen Völkerrecht und Landesrecht (z. B. Staatshaftung bei Nichterfüllung internationaler Vertragspflichten) entspricht bei umfangreichen Integrationsabkommen auch nicht den Integrationsbedürfnissen, und die mit der Wirtschaftsintegration verbundene regionale Kontrolle und rechtliche Überlagerung der nationalen Rechtsordnung impliziert wesentliche Änderungen im Landesrecht (z. B. Beschränkung der Parlamentskompetenzen). Diese Besonderheiten lassen jedoch noch nicht den Schluß auf einen „supranationalen" Charakter des Integrationsrechts der Entwicklungsländer $\mathrm{zu}$, da statt „supranationaler“ Rechtstechniken (z. B. Vorrang des Integrationsrechts, gemeinschaftliche Rechtsprechungsorgane) im Einzelfall auch traditionelle Methoden (Transformationserfordernis, lex posterior-Regel zugunsten späteren Landesrechts, Einstimmigkeitsprinzip) ausreichen und bei lockeren Freihandelsvereinbarungen wegen ihrer relativ geringen handelspolitischen Bedeutung dem Willen der Vertragspartner mehr entsprechen können.

Die Ratifizierungsverfahren waren bei den afrikanischen Integrationsabkommen unterschiedlich. Art. 65 UDEAC-Vertrag schreibt die Ratifizierung des gesamten Vertrages in Übereinstimmung mit der nationalen Verfassungspraxis der Vertragsstaaten und die Hinterlegung der Ratifizierungsurkunden vor. Der EAC-Vertrag trat dagegen gemäß Art. 91 ohne Ratifizierung in Kraft. In Art. 91/1 heißt es lediglich: „Each of the Partner States undertakes to take all steps within its power to secure the enactment and the continuation of such legislation as is necessary to give effect to this Treaty, and in particular:

(a) to confer upon the Community the legal capacity and personality required for the performance of its functions; and

(b) to confer upon Acts of the Community the force of law within its territory". Der EAC-Vertrag wurde daher von keiner Vertragspartei vollständig ratifiziert, sondern die Vertragsstaaten haben in übereinstimmenden Durchführungsgesetzen die einzelnen Vertragspflichten erfüllt und dabei das Verhältnis zwischen Integrationsrecht und Landesrecht geregelt. Die von der Ostafrikanischen Gesetzgeben- 
den Versammlung erlassenen und von den Staatspräsidenten der drei Mitgliedstaaten bestätigten (vgl. Art. 59 EACV) Gesetze gelten danach vom Tage ihrer Verkündigung im EAC-Amtsblatt als Teil des Landesrechts und gehen auch späteren Landesgesetzen vor, sofern letztere nicht ausdrücklich ihren Vorrang vor einem bestimmten EAC-Gesetz statuieren. Dieser die nationale Parlamentssouveränität sichernde Vorbehalt sollte die landesrechtliche Berücksichtigung lokaler Besonderheiten zulassen und wurde bis 1971 nur einmal im Zusammenhang mit dem „Tanzania-Zambia Railway Authority Agreement Act 1968“ seitens Tansania angewandt ${ }^{23}$. Für zivil- und strafrechtliche Streitigkeiten im Zusammenhang mit den EAC-Gesetzen sind regelmäßig in erster Instanz die nationalen Gerichte und in letzter Instanz das Ostafrikanische Berufungsgericht (vgl. Art. 81 EACV) zuständig. Ein Vorrang der EAC-Gesetze vor dem nationalen Verfassungsrecht wurde im Fall „The EAC v. The Republic of Kenya“ vom High Court of Kenya abgelehnt ${ }^{24}$. Der UDEAC-Vertrag wurde im Gegensatz zum EAC-Vertrag vollständig ratifiziert. Für die Entscheidungen der 2 Exekutivorgane sehen Art. 10, 18 UDEACV ausdrücklich die unmittelbare Wirkung im Recht der Mitgliedstaaten vor: „They shall be legally enforceable in the Member States one full day after the arrival of the Official Gazette of the Union in the capital of each Member State". Der in Art. 9, 17 UDEACV umschriebene Bereich der gemeinschaftlichen Rechtssetzungskompetenzen bezieht sich dabei auf nahezu die gesamte Zoll-, Fiskal- und Wirtschaftspolitik. Das Verhältnis zwischen Integrationsrecht und späterem, widersprechenden Landesrecht ist mangels vertraglicher Regelung und gerichtlicher Entscheidungen zwar nicht zweifelsfrei. In der Literatur wird jedoch ein den ausgeübten, gemeinschaftlichen Rechtsetzungskompetenzen korrespondierender Verlust der nationalen Kompetenzen und damit ein Vorrang des Gemeinschaftsrechts gegenüber einer nationalen „lex posterior" bejaht ${ }^{25}$.

Das Zollunion-Abkommen zwischen Lesotho, Betschuana, Swaziland und Südafrika wurde ohne Beteiligung der Parlamente aufgrund der nationalen Zollgesetzgebung allein von der jeweiligen Exekutive abgeschlossen und hat eine asymmetrische, einseitig auf den dominierenden Wirtschaftspartner Südafrika ausgerichtete Rechtsstruktur, insofern die Durchführungsgesetze von Swaziland, Betschuana und Lesotho die einseitige Übernahme der südafrikanischen Zolltarife und Zollgesetze vorsehen.

Die Verfassungen der meisten lateinamerikanischen Staaten setzen die „Transformation" internationaler Rechtsnormen für ihre Geltung im Landesrecht voraus. Die lateinamerikanischen Integrationsverträge wurden daher - mit Ausnahme des subregionalen Cartagena-Abkommens zur Gründung des Andenmarkts - regelmäßig in allen Vertragsstaaten vom Parlament gebilligt und förmlich ratifiziert. Hinsichtlich des sekundären Integrationsrechts wird dagegen - sofern es sich nicht schon um Integrationsnormen mit „self-executing“-Charakter handelt oder, wie in der

23 Vgl. hierzu: Akiwumi, RdC, Colloquium 1971, S. 80, 450 f., der mit Recht die "tenuous foundations“ (S. 82) der EAC betont: "to some extent its laws and their enforcement are left in the hands of the Partner States. Amity between the Partner States in this regard is of the utmost importance in the preservation and effectiveness of the laws of the EAC “ (S. 84). Zur neueren, politisch bedingten rechtlichen Desintegration in der EAC vgl. Afrika Forum 1973, S. $272 \mathrm{f}$

24 Siehe: Akiwumi, S. $83 \mathrm{f}$.

25 Akiwumi, S. 85 ("The 'supranationality' of the laws of the UDEAC seems clearly exemplified“), S. 449 ("The relationship between integration law and domestic law in the Central African Customs and Economic Union is very similar to that in the EEC“). Aus Art. 10, 18 UDEACV könnte dabei - parallel zu der im Art. 189 EWGV enthaltenen "Qualifizierung“ des Europäischen Gemeinschaftsredits - ein "supranationaler" Charakter des UDEAC-Rechts abgeleitet werden. 
Resolution 24 des CACM-Exekutivrats ${ }^{26}$, die unmittelbare Anwendbarkeit ausdrücklich vorgesehen ist - meist ein vereinfachtes Inkorpierungsverfahren allein durch Maßnahmen der Exekutive angewandt. Beim subregionalen, in das Integrationsrecht der LAFTA eingebetteten ${ }^{27}$ Cartagena-Abkommen wurde wegen der vorangegangenen parlamentarischen Billigung des LAFTA-Abkommens in Columbien, Chile und Peru ebenfalls auf eine erneute parlamentarische Billigung verzichtet und der Integrationsvertrag allein von der Exekutive abgeschlossen.

Die Rangfrage zwischen Integrationsrecht und Landesrecht ist mangels ausreichender Rechtsprechungspraxis für die meisten lateinamerikanischen Länder nicht eindeutig zu beantworten. In der Rechtsliteratur wird das Integrationsrecht dem Völkerrecht gleichgestellt und sein Vorrang gegenüber früherem Landesrecht mit dem Prinzip „lex posterior derogat legi priori“ und gegenüber späterem Landesrecht mit dem Prinzip "pacta sunt servanda“ begründet ${ }^{28}$. Eine solche Anwendung der völkergewohnheitsrechtlichen "pacta sunt servanda" - Regel für den innerstaatlichen Bereich ist jedoch keineswegs zwingend ${ }^{29}$. Zurückhaltung scheint auch deshalb am Platz, weil die Rechtswirkungen des sekundären Integrationsrechts in den Integrationsabkommen durchweg unpräzise oder gar nicht geregelt sind und dieselben lateinamerikanischen Autoren das Integrationsrecht wegen der Vorliebe der Regierungen für politische statt rechtlicher Lösungen als „system without any hierarchy of norms" kritisieren: „as the consensus of government is the principle point, decisions will have a higher hierarchy even in regard to treaty provisions so long as they are more coincident with the political purpose of government in attaining a certain goal" 30 . Hinsichtlich des CACM und des Andenmarkts scheint sich neuerdings jedoch die Rechtsansicht durchzusetzen, daß die gemeinschaftlichen Rechtssetzungskompetenzen zum Verlust der entsprechenden nationalen Kompetenzen führen und dadurch der Vorrang des Integrationsrechts eindeutig begründet wird.

Hinsichtlich der Rechtsangleichung, dem wohl wichtigsten Instrument der wirtschaftlichen Integration, sehen die lateinamerikanischen Integrationsabkommen keine kohärente Rechtsangleichungspolitik wie z. B. in Art. $100 \mathrm{ff.} \mathrm{EWGV} \mathrm{vor,} \mathrm{und}$ bislang wurden nur isolierte Harmonisierungsmaßnahmen durchgeführt. Der EACVertrag und der UDEAC-Vertrag sehen demgegenüber für die Ostafrikanische Gesetzgebende Versammlung bzw. für die UDEAC-Exekutivorgane umfangreiche Rechtssetzungsbefugnisse und in Art. 16, 19, 29/b EACV eine Harmonisierung des Steuer- und Handelsrechts vor. - Für die Vertragsänderungsverfahren, die gemeinschaftlichen "treaty making powers" oder für die Assoziation oder den Beitritt dritter Staaten enthalten die einzelnen afrikanischen und lateinamerikanischen Integrationsabkommen im übrigen meist unterschiedliche Regelungen.

\footnotetext{
26 „Resolutions... shall enter into force in each country eight days after the Permanent Secretariat has notified governments of such resolutions communicating to them the pertinent act".

$27 \mathrm{Vgl}$. Art. 110 Cartagena-Abkommen: "This Agreement shall remain in force as long as the engagements that are acquired in the general framework of the "Treaty of Montevideo do not surpass those that are here estabished"; Art 114: "Provisions of this Agreement shall not affect rights and obligations resulting from the Treaty of Montevideo and the Resolutions of LAFTA, which will be applied in a supplementary form".

28 Orrego Vicuña (s. o.), S. $148 \mathrm{f}$

29 Vgl. z. B. K. Doehring, Die allgemeinen Regeln des völkerrechtlichen Fremdenverkehrs und das deutsche Verfassungsrecht, 1963, S. $129 \mathrm{ff} ., 135 \mathrm{ff}$.

30 Orrego Vicuña, S. $405 \mathrm{f}$.
} 


\section{3. Institutionelle Probleme}

Die Wirksamkeit des wirtschaftlichen Integrationsrechts hängt erfahrungsgemäß entscheidend von seinen institutionellen Regelungen ab. Während C. F. Ophüls ${ }^{31}$ hinsichtlich der Europäischen Gemeinschaften die institutionellen Regelungen als das wesentlich Neue und als einen der Hauptgründe für den Erfolg der europäischen Wirtschaftsintegration bezeichnet hat, haben lateinamerikanische Experten wie H. Gros Espiell ${ }^{32}$ den Mißerfolg der LAFTA u. a. mit dem Fehlen eines das Gemeinschaftsinteresse vertretenden, unabhängigen Gemeinschaftsorgans sowie eines regionalen Rechtsprechungsorgans erklärt. In dem um das Vermeiden der negativen Erfahrungen der LAFTA bemühten, subregionalen Andenmarkt-Abkommen ist daher nach dem Vorbild des EWGV die "Junta" als ein unabhängiges, das regionale Interesse repräsentierendes und damit über die traditionellen Formen multilateraler Diplomatie hinausgehendes Gemeinschaftsorgan errichtet sowie mit einem dem Art. 149 EWGV nachgebildeten Vorschlagsrecht gegenüber dem aus Regierungsvertretern zusammengesetzten Hauptentscheidungsorgan („Kommission“) ausgestattet worden. Die durch die Erfahrungen der EG-Kommission oder auch des UNCTAD-Sekretariats bestätigte Notwendigkeit unabhängiger Initiativ- und starker Gemeinschaftsorgane ist für die Wirtschaftsintegration zwischen Entwicklungsländern um so offensichtlicher, weil hier die Handelsliberalisierung notwendig durch eine gemeinsame Entwicklungsplanung mit gemeinschaftlichen Planungsinstanzen ergänzt werden muß und wegen des Fehlens gemeinschaftlicher Rechtsprechungsorgane der vorherige Interessenausgleich mit Hilfe unparteiischer Gemeinschaftsorgane um so wichtiger ist. Die Bereitschaft der Entwicklungsländer zur Errichtung "supranationaler" Gemeinschaftsorgane ist jedoch außerhalb der EAC, der UDEAC und des Andenmarktes gering und wird u. a. durch den of geringen handelspolitischen Anteil des regionalen Präferenzzonenhandels am gesamten Außenhandel dieser Länder (z. B. weniger als zwei Prozent bei den Maghrebländern und den Mitgliedsländern der Regional Cooperation for Development), durch den Mangel an Technokraten und „know how“, die hohen Kosten solcher Gemeinschaftsorgane und durch das nationale Souveränitätsdenken behindert.

Die lateinamerikanischen Integrationsabkommen sehen mit Ausnahme des Cartagena-Abkommens regelmäßig eine institutionelle Struktur traditionellen, zwischenstaatlichen Charakters vor: die Hauptentscheidungsorgane setzen sich aus Regierungsvertretern zusammen; die Organkompetenzen sind oft nur vage und unpräzise festgelegt, zumal die einzelnen Mitgliedstaaten auch bei Mehrheitsbeschlüssen eine Vetomöglichkeit haben (Art. 38 LAFTAV, Art. XXI CACM) und der dominierende Einfluß der Regierungen dadurch sichergestellt ist; das Übergewicht der nationalen Interessen wird durch die meist schwache Stellung der in den Integrationsabkommen regelmäßig vorgesehenen Sekretariate ${ }^{33}$ nicht ausgeglichen; eine Legalitätskontrolle durch gemeinsame Gerichte fehlt durchweg, und auch die Möglichkeiten für wirksame, gemeinschaftsrechtliche Sanktionen sind meist gering. Die seit 1965 im Rahmen der LAFTA unternommenen Bemühungen um institutionelle Reformen zugunsten eines angemessenen Gleichgewichts zwischen regionalen und nationalen Interessen blieben ebenfalls wenig erfolgreich: die Mitglieder der 1965 durch Resolution 118 errichteten, das regionale Interesse repräsentierenden

31 C. F. Ophüls, in: Einführung in die Rechtsfragen der europäischen Integration, 1969, S. 15 ff.

32 In: RdC, Colloquium 1971, S. 551.

33 In der LAFTA gilt das Sekretariat (vgl. Art. 41) nicht einmal als „Organ“ der LAFTA, vgl. Art. 33. 
„Technische Kommission“ wurden bislang nicht ernannt; das am 12. 12. 1966 unterzeichnete Protokoll über die Errichtung eines Ministerrats trat nicht in Kraft; die zunächst angestrebten, institutionellen Kontakte zwischen den nationalen Parlamenten wurden nicht verwirklicht; das in dem am 2. 9. 1967 unterzeichneten Protokoll enthaltene Streitbeilegungsverfahren sieht politische und in letzter Instanz schiedsgerichtliche Verfahren zur Beilegung zwischenstaatlicher Streitigkeiten vor, ohne daß ein ständiges Gericht errichtet, der direkte Zugang für Private eröffnet oder die Möglichkeit von Entscheidungen ex aequo et bono und von Rechtsgutachten des Schiedsgerichts zugelassen wird; dem Schiedsgericht wurde darüber hinaus zum Schutze der politischen LAFTA-Organe vor einer "judicial legislation“ paradoxerweise eine Interpretation des LAFTA-Vertrags ausdrücklich untersagt, womit eine kontinuierliche Rechtsprechung und wirksame gerichtliche Streitbeilegung unmöglich gemacht sind ${ }^{34}$.

Für die UDEAC und die EAC sind die aus den Staatspräsidenten zusammengesetzten, nahezu omnipotenten und daher schnelle und wirksame Entscheidungen ermöglichenden Exekutivorgane kennzeichnend. Im Hinblick auf die umfangreichen Kompetenzen des EAC-Sekretariats (Art. 31), die Einsetzung ständiger Ostafrikanischer Minister zur Wahrung des Gemeinschaftsinteresses (Art. 49 ff.) und der ansatzweise mit der Schaffung der Ostafrikanischen Gesetzgebenden Versammlung (Art. 56 ff.) sowie dreier ostafrikanischer Gerichte (Art. 32, 80, 85) verwirklichten Gewaltenteilung dürften die institutionellen Regelungen des EAC-Vertrags zu den fortschrittlichsten aller Integrationsabkommen der Entwicklungsländer gehören. Das Zollunion-Abkommen zwischen Betschuana, Lesotho, Swaziland und Südafri$\mathrm{ka}$ sieht demgegenüber als einziges gemeinsames Organ lediglich eine aus Vertretern der Vertragsparteien zusammengesetzte Kommission mit allein konsultativen Aufgaben vor, ähnlich wie auch andere "Nord-Süd-Präferenzabkommen“ z. B. zwischen der EWG einerseits und Spanien oder den Maghrebländern andererseits nur einen Ausschuß bzw. Assoziationsrat mit im wesentlichen beratenden Funktionen vorsehen.

Außerhalb der EAC sind die exekutiven und legislativen Befugnisse regelmäßig nicht institutionell getrennt. Die Ostafrikanische Behörde, der UDEAC-Rat und auch die Exekutivorgane der meisten lateinamerikanischen Integrationssysteme entscheiden einstimmig oder sehen bei Mehrheitsbeschlüssen eine Vetomöglichkeit für überstimmte Mitgliedstaaten vor. Institutionelle Mechanismen für eine wirksame Interessenbalancierung werden dabei mit Recht für wichtiger gehalten als Gewaltenteilung und Mehrheitsbeschlüsse, da die für jede Wirtschaftsintegration erforderliche Solidaritätsbasis besonders zwischen Entwicklungsländern eine möglichst weitgehende Übereinstimmung bedingt. Das - ausgenommen die EAC regelmäßige Fehlen ständiger, gemeinsamer Integrationsgerichte zur Gewährleistung der für die Rechtssicherheit und Kalkulierbarkeit der Integrationsrisiken notwendigen Rechtsschutzverfahren, der einheitlichen Interpretation des Integrationsrechts und einer gerichtlichen Legalitätskontrolle der nationalen und gemeinschaftlichen

\footnotetext{
34 Vgl. H. Gros Espiell, S. 551 („Ceci fut évidemment une grave erreur juridique, car l'application d'une norme n'est jamais possible sans son interprétation préalable"), und F. Orrego Vicuña, S. 133 ("But this absurd situation goes even further. According to Art. XXXI of the American Treaty for Pacific Settlement, Contracting Parties recognize as compulsory ipso facto and without special agreement, in relation to any other American State, the jurisdiction of the International Court of Justice in all legal disputes. . It could well happen that two parties to this treaty and, at the same time, parties to the Treaty of Montevideo confronting one another in a dispute of interpretation of the latter, may find their settlement through the International Court of Justice and not through LAFTA's own mechanism“.), in: RdC, Colloquim 1971.
} 
Behörden ist auch unter diesem Aspekt der Interessenbalancierung sowie der Entpolitisierung der von den Gemeinschaftsorganen ausgeübten Kontrolle über nationale, wirtschaftspolitische Maßnahmen nachteilig. In der EWG hat sich jedenfalls die Rechtsprechung des Europäischen Gerichtshofs (EuGH) als für den Erfolg der Wirtschaftsintegration unentbehrlich erwiesen, und die mutige "judicial legislation“ des EuGH hat ihn zu einer der progressivsten Kräfte zugunsten einer supranationalen Wirtschaftsintegration gemacht. 
"Fearless-Proposals" of the Labour Government to the Anglo-Rhodesian agreement of 1971. It is shown that both the Labour and the Conservative governments, which refused to resort to military intervention for enforcing its imperial legal claim, with the application and timing of their "Six Principles" agreed to a settlement that would have brought majority rule in about 100 years' time, according to pessimistic estimates, and even on very optimistic assumptions no sooner than in 50-60 years' time. The Pearce Report documented the rejection of this agreement between two white governments by a overwhelming majority of the Africans. After the failure of international sanctions due to the complicity of white regimes in Southern Africa and international economic interest only the Liberation Movements could defeat the internal colonialism in Rhodesia.

\section{The Shifta-War in Kenya}

\section{By Volker Matthies}

The most immediate and serious security threat to independent Kenya was the guerilla warfare of the Somali shifta fighters in the north-eastern part of the country. Economically and socially neglected by the British colonial administration, the Somali in Kenya were highly suspicious of the central government. Moreover, the Somali were influenced to a high degree by tribal feelings and nationalist emotions, which were based on an ethnic-cultural identity of all Somali people living in the Horn of Africa and which aimed at secession from Kenya and at political union with the neighbouring Republic of Somalia. Thus a growing number of Somali in Kenya felt no strong loyalty to the new state in which they happened to live but instead considerable sympathy with the Somali Republic, and they took their resentment towards the Bantu-dominated Kenya government to the stage of an armed insurgent secessionist movement. Although the shifta warfare was truly locally inspired, it gained increasingly support from the Republic of Somalia. For the government of Kenya the shifta-guerilla was an essentially tribalist movement which threatened the territorial integrity and political sovereignty of the state. Since Kenya lacked internal cohesion and a deeply rooted nationhood, any surrendering to secessionist pressure was dangerous. To allow one etnic or tribal group to secede might lead to secessionist or separationist tendencies elsewhere and might add new problems to the difficult task of nation-building. Thus the government of Kenya has been involved since 1963 on an increasing scale in a costly anti-guerilla warfare to eliminate the shifta threat. After four years of fighting the government was successful in creating military and political control in the Somali area of Kenya.

Theory and Comparative Legal Aspects of Economic Integration among Developing Countries

\section{By ERnst U. Petersmann}

Regional economic integration by means of economic unions, common markets, customs unions, free trade areas, partial preferential agreements or project arrange- 
ments is one of the essential features of the present world trading system. As most Less Developed Countries (LDCs) are too small to be able to create modern, internationally competitive industrial structures within their national frameworks, regional economic integration among LDCs is now widely accepted as a sine qua non for effective industrialisation in most LDCs and as a means to strengthen regional solidarity as well as the international bargaining position of LDCs. Contrary to the steadily increasing literature on economic aspects of regional integration among LDCs, there are only few comparative legal studies as to the law of the some 20 major regional economic groupings among LDCs.

Chpt. 1 on "Regional economic integration among LDCs: comparative economic experiences" summarizes the conclusions of the many UNCTAD-, OECD- and other expert-reports as to the economic rationale, potential benefits, pre-conditions for successful implementation, main impediments and up-to-now experiences of economic integration schemes among LDCs. Chpt. 2 on "A legal integration theory?" sketches the development of customs union theories and political integration theory in order to inquire into the pre-conditions for a legal integration theory based on comparative legal experiences with the legal systems of regional economic groupings; as law is a necessary instrument of economic integration, comparative legal analysis of successful legal integration techniques may prove to be helpful for increasing efficiency of economic integration among LDCs (cf. the successful application of EECs institutional techniques within the Andean Common Market-Agreement e. g. as to the mechanism of "propositions" from the regional "Junta" to the ministerial "Commission"). Chpt. 3 on "Comparative legal aspects of economic integration among LDCs" describes and compares the legal regulation of three problem areas common to the law of all regional economic groupings: integration law and economic planning (e.g. integration treaties as "living constitutions" and "traités cadre"; escape clauses to adopt to economic dynamism; preferential measures for least developed member states to ensure an equitable distribution of benefits; problems as to the compatibility of "secondary" with "primary" integration law etc.); the interplay and symbiosis between regional integration law and national law; institutional structures and techniques of the various economic groupings. 